\title{
Quadrilateral Modelling and Robust Control of a nonLinear Piezoelectric Cantilever
}

\author{
Micky Rakotondrabe, member, IEEE, Yassine Haddab and Philippe Lutz, member, IEEE
}

\begin{abstract}
Piezocantilevers are commonly used for the actuation of micromechatronic systems. These systems are generally used to perform micromanipulation tasks which require high positioning accuracy. However, the nonlinearities, i.e. the hysteresis and the creep, of piezoelectric materials and the influence of the environment (vibrations, temperature change, etc.) create difficulties for such a performance to be achieved. Various models have been used to take into account the nonlinearities but they are often complex. In this paper, we study a one degree of freedom piezoelectric cantilever. For that, we propose a simple new model where the hysteresis curve is approximated by a quadrilateral and the creep is considered to be a disturbance. To facilitate the modelling, we first demonstrate that the dynamic hysteresis of the piezocantilever is equivalent to a static hysteresis, i.e. a varying gain, in series with a linear dynamic part. The obtained model is used to synthesize a linear robust controller, making it possible to achieve the performances required in micromanipulation tasks. The experimental results show the relevance of the combination of the developed model and the synthesized robust $H_{\infty}$ controller.
\end{abstract}

Index Terms-Piezoelectric devices, hysteresis and creep, quadrilateral approximate model, robust control, micromanipulation.

\section{INTRODUCTION}

$\mathbf{P}$ Iezoelectric materials are commonly used for the actuation of microsystems and microrobots. This is due to their good deformation/force ratio, high resolution and low response time. One of their major applications in the microworld is the actuation of microgrippers, as in [1] [2] [3]. A piezoelectric microgripper (Fig. 1) is generally made up of two piezocantilevers (piezoelectric cantilevers). It is used to perform micromanipulation tasks (manipulation of microobjects). According to the application, the cantilevers may be controlled in position and/or in force.

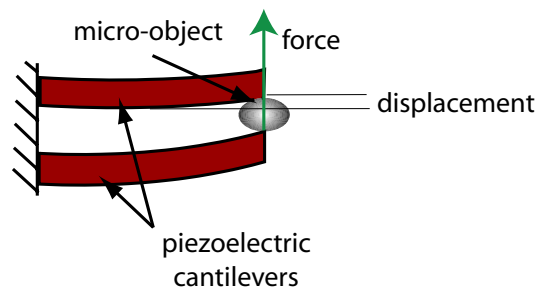

Fig. 1. A piezoelectric microgripper made up of two piezocantilevers.

FEMTO-ST Institute, UMR CNRS 6174 - UFC / ENSMM / UTBM Automatic Control and Micro-Mechatronic Systems depart., AS2M

24, rue Alain Savary

25000 Besançon - France

mrakoton@femto-st.fr, \{yhaddab,plutz\}@ens2m.fr

Paper type: regular paper.First submission: June 2006. Accepted: Mars 2008
In micromanipulation tasks, the displacement at the tip,

i.e. the deflection, of one piezocantilever is often lower than a few tens of microns while the accuracy is submicrometric. Such systems are very sensitive to the environment. For example, environmental vibrations can produce displacements greater than the reference deflections. These environmental vibrations are often caused by surrounding electrical devices and instruments. To perform a micromanipulation task, these disturbances must be taken into account during the design of the controllers.

Due to their small size, piezocantilevers can be damaged during micromanipulation tasks. In this case, they have to be replaced. The controller must then ensure the stability and the performance in spite of the exchange of piezocantilevers, as small geometrical differences always exist between the piezocantilevers. As the size of the piezocantilever in its entirety is relatively small (a few millimeters), such a difference extensively influences the models parameters.

One of the major limitations for piezoelectric actuators to achieve high tracking accuracy is due to their inherent nonlinear behavior. It has been shown that when the deflexion $\delta$ of the cantilever becomes large, generally higher than $15 \%$ of the maximum field strength [4], hysteresis and creep phenomena arise and the performances, notably the accuracy, of the piezoactuators are decreased. To succeed a micromanipulation task and maintain the required performances, the influences of the hysteresis and the creep must be rejected either with open loop or closed-loop techniques.

In the open loop techniques, two linearization methods are used for piezoelectric materials: the charge compensation and the model compensation. As the relation between the applied charge and the deflection is linear, the charge compensation consists in converting the voltage into charge and controlling the piezocantilever through the latter [5]. In the model compensation, the principle is to precisely model the nonlinearities and place the corresponding inverse model in series with the real system. The accuracy of the control depends on the accuracy of the nonlinear model and its inverse. However, open loop techniques are not suitable for disturbed systems and closed-loop techniques should be used.

Concerning closed-loop techniques, feedforward-feedback and feedback methods are used. The feedforward-feedback method consists in placing an inverse hysteresis model in the feedforward loop to cancel the hysteresis, and design a linear feedback controller to improve the performances. In the feedback method, a linear approximate model is used to model 
the nonlinearities and to design a linear controller.

For both techniques, various nonlinear models and linear approximate models were proposed. For example, a logarithmic function [6] or the Maxwell model [7] [8] were used to model the creep. To model the hysteresis, the Preisach model is the most accurate [9] [10] [11]. However, due to its complexity, the real time implementation of the Preisach model is difficult [12]. The same problem also exists in other precise models [13], such as the Maxwell resistive capacitor model [14] and the Bouc-Wen model (tuning voltage based model) [15] [16]. Consequently, linear approximate models were proposed. They are the polynomial model [18] [13] and the variable gain - variable phase model [19]. In the polynomial model, polynomial functions are used to approximate the ascending curve and the descending curve of the hysteresis. The control schemes based on this approximation are very easy to implement but as the coefficients of the polynomials are experimentally determined with a sine input at constant frequency and amplitude, the magnitude and rate dependence of the hysteresis were not considered [19] [20] [21]. Finally, the variable gain - variable phase model consists in having a linear model with a time-delay. In this approximation, the gain depends on the amplitude of the input while the timedelay depends on its frequency. As the approximate model only considers the hysteresis phenomenon, the creep is not taken into account. A detailed survey of the different control techniques for piezoactuators can be found in [22].

In this paper we present a simple model easy to identify for a one degree of freedom (dof) piezoelectric cantilever. This model, which does not need to be implemented (as in the inversion compensation method), is used to synthesize low power and low time consuming controllers. The proposed model takes into account the hysteresis and the creep. In this model, the model is approximated by a linear model subjected to uncertainty and with a varying static gain. Before detailing the new approximation, we first demonstrate that the hysteresis only affects the gain of the voltage-deflection relation. Consequently, as the hysteresis is rate-independent, the coefficients of the proposed approximate model are independent of the frequency of the input voltage. However, it will be shown that the static gain is subjected to uncertainty. So, to ensure the performances required in the micromanipulation stated above, a robust $H_{\infty}$ controller is proposed at the end of the paper.

\section{HYSTERESIS AND CREEP IN PIEZOELECTRIC CANTILEVERS}

In this section, we show experimentally that the hysteresis in a piezocantilever only affects the static domain. Such hysteresis is called static hysteresis or rate-independent hysteresis, as its shape is independent of the input signal frequency [23]. The fact that the piezocantilever hysteresis is static is of great interest because the approximate model is independent of the input signal frequency. Before that, we analyze the hysteresis and the creep in a piezocantilever.

For the experiments, a unimorph piezocantilever made up of a PIC151 piezolayer and a copper layer is used. The characteristics of the cantilever are presented in Table I.
The experimental setup (Fig. 2) comprises a computer with Matlab-Simulink software, a DSpace real-time board, a voltage amplifier and a laser sensor with an accuracy of $0.5 \mu \mathrm{m}$ (from KEYENCE).

TABLE I

Geometrical parameters of the piezocantilever.

\begin{tabular}{|l|l|}
\hline \hline \multicolumn{2}{|c|}{ Dimensions: } \\
\hline Length & $15 \mathrm{~mm}$ \\
\hline Width & $2 \mathrm{~mm}$ \\
\hline Total thickness & $0.3 \mathrm{~mm}$ \\
\hline PIC151 thickness & $0.2 \mathrm{~mm}$ \\
\hline Copper thickness & $0.1 \mathrm{~mm}$ \\
\hline
\end{tabular}

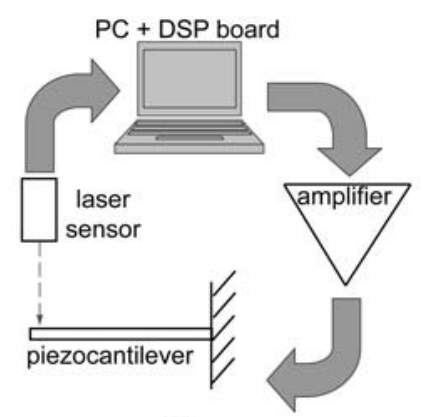

(a)

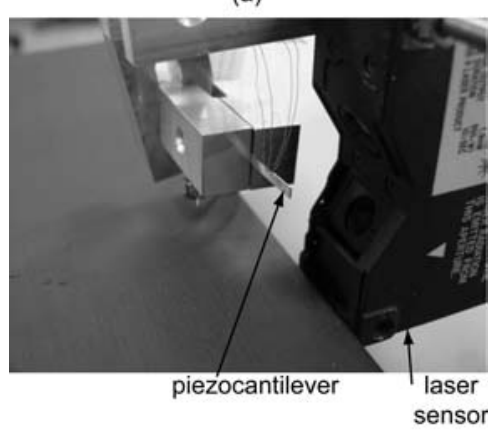

(b)

Fig. 2. The experimental setup.

\section{A. Description of the piezocantilever behaviour}

Let Fig. 3 represent a piezocantilever under electrical and mechanical stimulations respectively $U$ and $F$. The piezocantilever may be a unimorph or a bimorph (made up of two piezolayers) piezoelectric cantilever.

The relation between the deflection $\delta$, the applied force $F$ and the voltage $U$ when the electromechanical part is nonlinear is as follows [26]:

$$
\delta(s)=s_{p} \cdot D(s) \cdot F(s)+\Gamma(U(s), s)
$$

where $s_{p}$ is the elastic constant, $D(s)$ is a dynamic part, $s$ the Laplace variable and $D(0)=1$. For the rest, the Laplace 


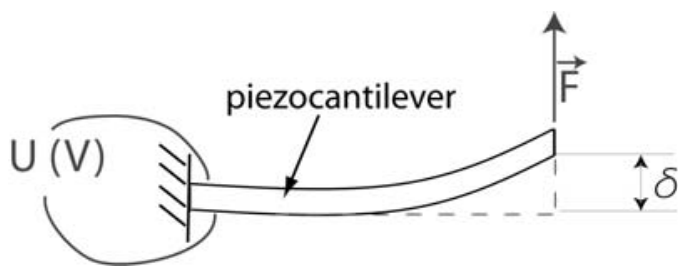

Fig. 3. A piezocantilever under an electrical and a mechanical excitations.

signals $\delta(s), U(s)$ and $F(s)$ are replaced by $\delta, U$ and $F$ except in the figures where the latter represents the signals in the temporal domain.

$\Gamma(U, s)$ is an operator that includes the hysteresis and the creep. Since this operator includes the hysteresis, it depends on the past and present values of $U$. In addition, it may depend on the frequency of $U$. This is why the notation of $\Gamma$ has both $U$ and $s$. Let us study the possible separation of the two nonlinearities inside $\Gamma(U, s)$.

The creep can be observed when a step voltage is applied to the piezocantilever (Fig. 4-a). The drift tends towards a finite value and its amplitude depends on the step amplitude. If we consider that the drift begins just after the transient part, we can write:

$$
\Gamma(U, s)=H(U, s)+C_{r}(U, s) \cdot e^{-T \cdot s}
$$

where $H(U, s)$ is the hysteresis operator, $C_{r}(U, s)$ is the creep operator and $T$ is the delay before the creep starts. However, many experiments have shown that the transient part of piezocantilevers is generally below $100 \mathrm{~ms}$ while its creep response time is more than $3 \mathrm{~min}$. The delay can then be neglected from the expression (Eq. 2) (Fig. 4-b). We obtain:

$$
\delta=s_{p} \cdot D(s) \cdot F+H(U, s)+C_{r}(U, s)
$$
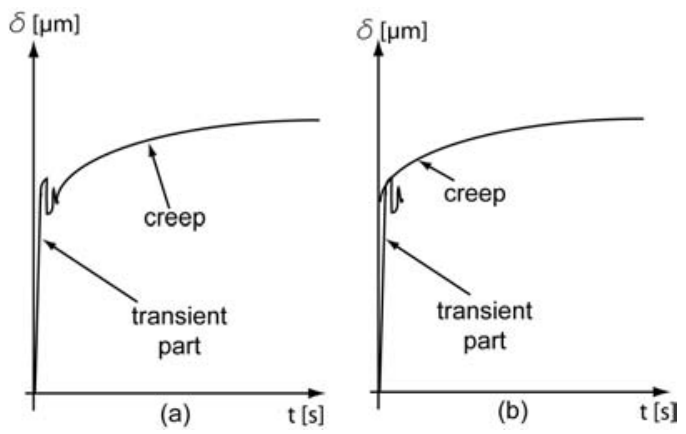

Fig. 4. Creep phenomenon.

In the expression (Eq. 3) and according to Fig. 4-a, the static value and the transient part of the electromechanical transfer is modelled inside the hysteresis term $H(U, s)$. So, the creep $C_{r}(U, s)$ can be considered as a disturbance. Hence, one of the aims of this paper is to approximate the hysteresis term by a simpler one. Before that, let us first demonstrate that $H(U, s)$ can be divided into two more simple terms.

\section{B. Analysis of the hysteresis $H(U, s)$}

To analyze the hysteresis, we apply a sine voltage input to the piezocantilever and no force is applied to its tip. While the amplitude of the input signal is constant $(=40 \mathrm{~V})$, different frequencies are used. The experimental results show that the shape of the hysteresis depends on the input signal frequency (Fig. 5). Such a hysteresis is called dynamic hysteresis or ratedependent hysteresis [23].
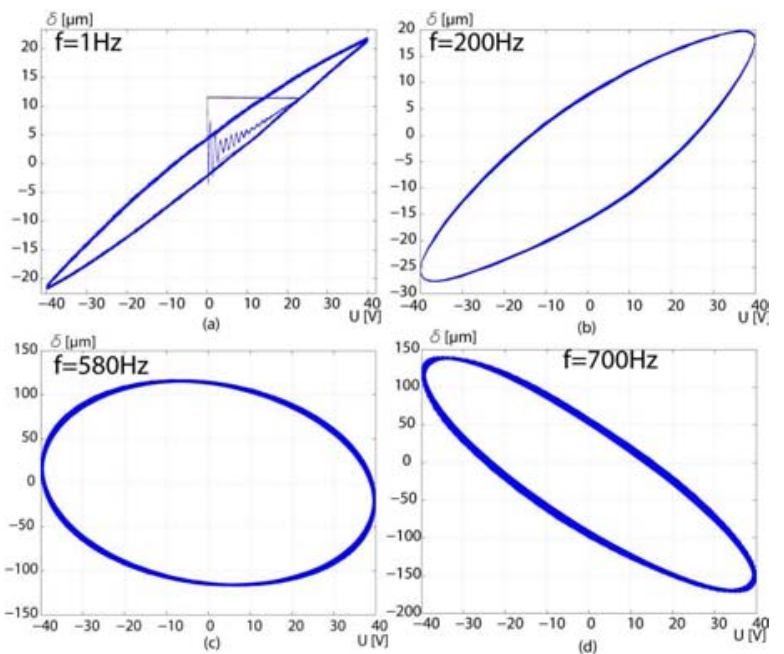

Fig. 5. The hysteresis of the piezocantilever is dynamic, i.e. rate-dependent.

An approximate model of a dynamic hysteresis should have parameters which are also rate-dependent. This will firstly increase the complexity of the model and therefore the complexity of the controller. To avoid that, we propose an easier equivalence of the dynamic hysteresis in this section. We show experimentally that the dynamic hysteresis $H(U, s)$ is equivalent to a static hysteresis $H_{i}(U)$ followed by the linear dynamical part $D(s)$ as shown in Fig. 6. A static hysteresis has a constant shape whatever the frequency of the input.

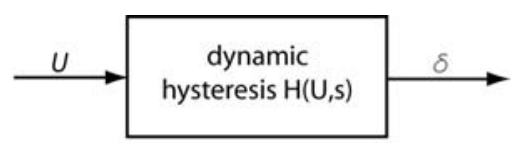

(a)

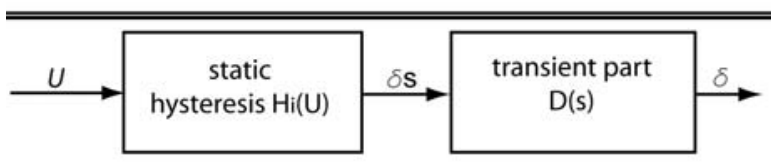

(b)

Fig. 6. The dynamic hysteresis is equivalent to a static hysteresis followed by a linear dynamic part.

The experimental steps are as follows.

- Step 1: We first show that the transient part of piezocantilever is independent of the amplitude of the input 
$U$. The transient part being given by a step response, we use a square signal with different values of amplitude during the experiments. The dynamic part $D(s)$ can then be identified.

- Step 2: Following Fig. 6-b, we simulate and plot the magnitude of $H_{i} . D(s)$. The aim is to compare the obtained magnitude with that of the piezocantilever which is experimentally performed. For both, we use a sine wave signal $U$ with different values of amplitude. If the two curves coincide, the simplification of the hysteresis such as $H(U, s)=H_{i}(U) \cdot D(s)$ is validated. However, we need a precise static hysteresis model $H_{i}$ for the simulation. For that, we use the Bouc-Wen static hysteresis model because of its accuracy. This model is only used to validate the simplification and will not be implemented or used to synthesize a controller.

- Finally, when the relation $H(U, s)=H_{i}(U) \cdot D(s)$ is validated, we propose to approximate the static hysteresis $H_{i}$ by a new approximate model: the quadrilateral approximation. The aim is to obtain a simple model which is easy to identify. This model is linear contrary to other models (Bouc-Wen, Preisach, etc.) which are nonlinear. However, the proposed model is less accurate than them, so we propose to synthesize a linear robust controller. The uncertainty is easy to derive in the proposed model.

\section{1) Step 1: independence of the transient part.}

We apply a square voltage $U$ with a given frequency ( $f=$ $0.3 \mathrm{~Hz}$ ) to the piezocantilever. Two amplitudes were used: $U=$ $40 \mathrm{~V}$ and $U=20 \mathrm{~V}$. The corresponding step responses are given in Fig. 7. Besides the creep phenomenon, we notice the similarity of the transient parts for all the responses.

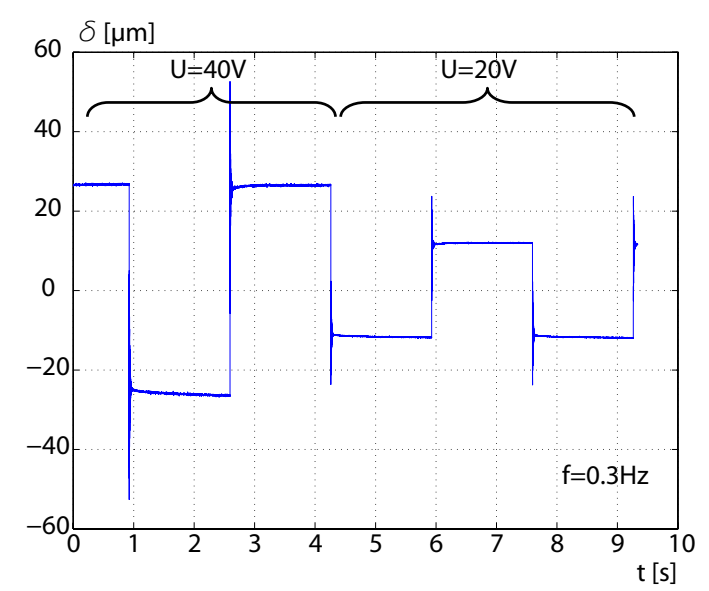

Fig. 7. Response of the piezocantilever when a square signal is applied. The different step responses are similar.

From the previous experiments, we conclude that the transient part is independent of the amplitude of the input. In addition, when a repeated step signal (i.e. a square signal) is applied, the transient parts are similar. It can be concluded that a dynamic part $D(s)$ can be separated from a static part which contains the static gain. Let us use the previous notation $D(s)$ for the transient part. To identify $D(s)$, any step response can be used (Fig. 8). It can be approximated by a second order model [2] [16]:

$$
D(s)=\frac{1}{a \cdot s^{2}+b \cdot s+1}
$$

where $a=4.722 \times 10^{-8} \sec ^{2}$ is the inertial coefficient and $b=1.304 \times 10^{-5} \mathrm{sec}$ is the viscous coefficient.

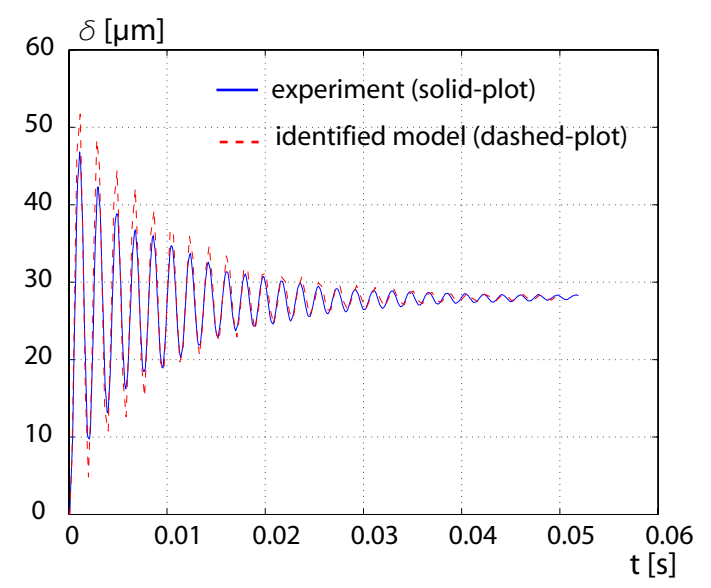

Fig. 8. Step response of the real system and of the dynamic model $D(s)$. The static gain of $D(s)$ has been scaled in order to allow the comparison.

In order to confirm that the piezocantilever can be modelled by the dynamic part $D(s)$ in series with a static part, a harmonic analysis is performed. For that, a sine voltage with $40 \mathrm{~V}$ of amplitude is applyed to the piezocantilever, i.e. to $H(U, s)$. the corresponding magnitude is plotted. After that, the magnitude of the identified $D(s)$ is plotted in the same graph ((Fig. 9)). After scaling the static gain of $D(s)$ in order to allow the comparison, it is shown that the two curves almost coincide with each other. However, in high frequency, i.e. above $10^{4}[\mathrm{rad} / \mathrm{s}]$, the slopes are different. That is due to the choice of the order of $D(s)$. Using a higher order of $D(s)$, the deviance of the magnitudes is minimized even in high frequency. For example, Fig. 9-star-plot shows the simulation of $D(s)$ with a sixth order model.

Finally, it is experimentally shown that the shape of the magnitude of $H(U, s)$ does not depend on the amplitude $U$. Fig. 10 shows that the magnitude obtained with an amplitude of $40 \mathrm{~V}$ is similar to the one with $20 \mathrm{~V}$ except the static gain. The difference between the static gains indicates the presence of a hysteresis inside the static part.

The harmonic analysis demonstrates that $H(U, s)$ is similar to $D(s)$ except for a static gain. As $D(s)$ is amplitudeindependent, the static gain should contain the hysteresis. 


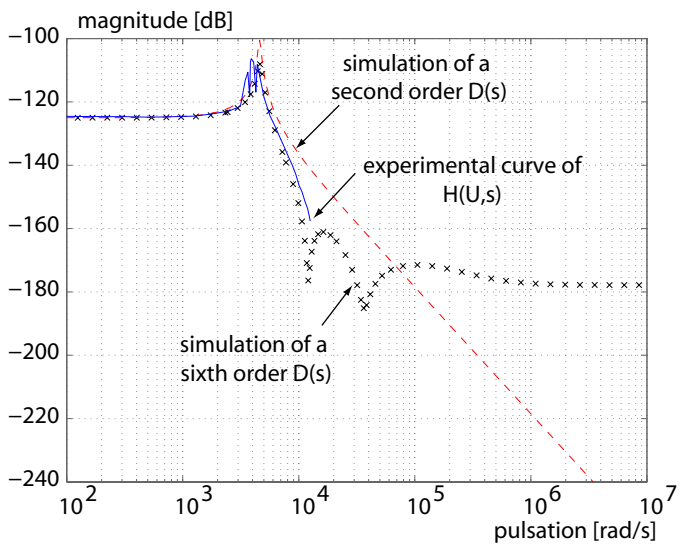

Fig. 9. Experimental magnitude of $H(U, s)$ and simulated magnitude of $D(s)$.

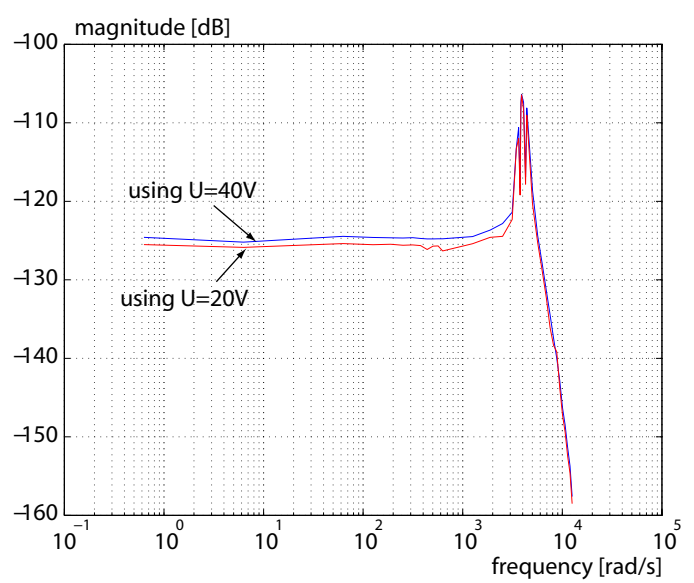

Fig. 10. Experimental magnitude of $H(U, s)$ using two different amplitudes.

This is a static hysteresis.

2) Step 2: comparison of the simulation $H_{i} \cdot D(s)$ and the harmonic experiment results.

In this step, we demonstrate that $H(U, s)=H_{i}(U) \cdot D(s)$, where $H_{i}(U)$ is a static hysteresis that represents the gain. For that, we compare the simulation of $\delta=H_{i}(U) \cdot D(s)$ with the experimental results of $H(U, s)$ in the $(U, \delta)$-plane. However, while $D(s)$ is given by (Eq. 4), the simulation requires a precise model of the static hysteresis $H_{i}(U)$. The Bouc-Wen hysteresis model has been chosen due to its accuracy. Applied to piezocantilevers, this model is represented by the following set of equations [16]:

$$
\begin{aligned}
& \delta=s_{p} \cdot F+\left(d_{p} \cdot \mathrm{U}-\mathrm{h}\right) \\
& \dot{h}=A^{b w} \cdot \dot{U}-B^{b w} \cdot|\dot{U}| \cdot h-\Gamma^{b w} \cdot \dot{U} \cdot|h|
\end{aligned}
$$

where:

- $s_{p}$ and $d_{p}$ are respectively the elastic constant and the piezoelectric constant,

- $F$ is the external force (null for the different experiments and simulations),

- $h$ is an internal variable,
- $U$ is the applied voltage,

- $A^{b w}$ is a parameter which determines the amplitude of the hysteresis,

- $B^{b w}$ et $\Gamma^{b w}$ are parameters which determine the shape of the hysteresis.

The model in the (Eq. 5) can be considered as static at least until $500 \mathrm{kHz}$ (checked by simulation) which is very high compared to the cut-off frequency of the whole system. The parameters of the model were identified using the values given in [16] that were adjusted. We obtain:

$$
\left\{\begin{array}{l}
d_{p}=1.0773 \times 10^{-6}[\mathrm{~m} / \mathrm{V}] \\
s_{p}=1.931 \times 10^{-3}[\mathrm{~m} / \mathrm{N}] \\
A^{b w}=6.064810 \times 10^{-7}[\mathrm{~m} / \mathrm{V}] \\
B^{b w}=0.00833\left[\mathrm{~V}^{-1}\right] \\
\Gamma^{b w}=0.00833\left[\mathrm{~V}^{-1}\right]
\end{array}\right.
$$

The experiments consist in applying a sine voltage to the piezocantilever and tracing the corresponding $(U, \delta)$-plane curve. Since the cantilever includes creep and hysteresis phenomena, the frequency of the sine input signal should be higher than $f_{c c}$ which is the cut-off frequency of the creep. With $f_{c c} \leq \frac{1}{2 \cdot \pi \cdot \tau_{c}} \approx 1 \mathrm{mHz}$ and $\tau_{c} \geq 3$ minutes is the response time of the creep. The plotted $(U, \delta)$-plane curve only corresponds to the hysteresis $H(U)$. Different values of frequency were used: $f=\{1 \mathrm{~Hz}, 300 \mathrm{~Hz}, 600 \mathrm{~Hz}, 900 \mathrm{~Hz}\}$ while the amplitude was $40 \mathrm{~V}$. The experiments and the simulation are shown in Fig. 11. The comparison results show clearly that the dynamic hysteresis is equivalent to a static hysteresis followed by a dynamic part: $H(U, s)=H_{i}(U) \cdot D(s)$.
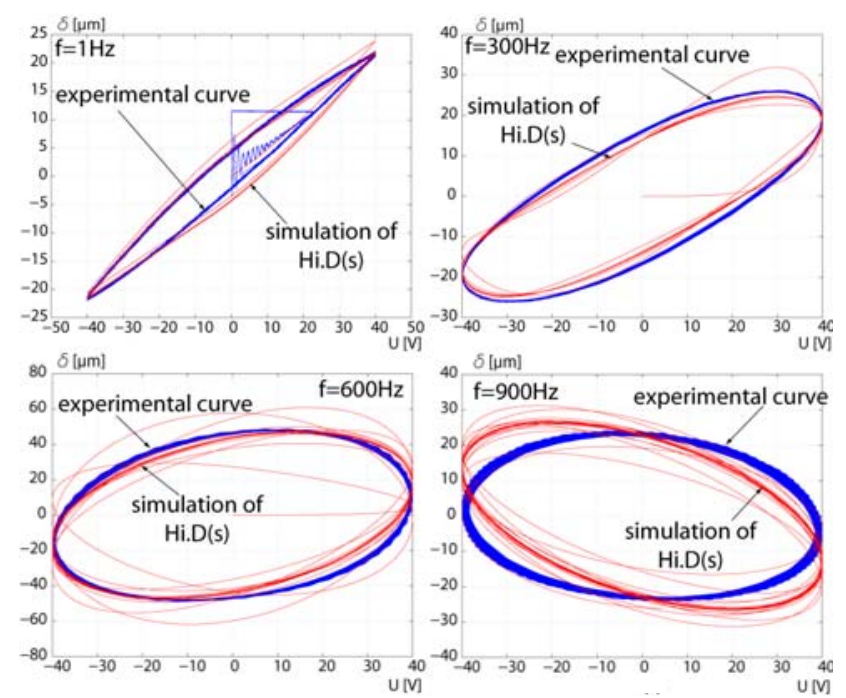

Fig. 11. Comparison of $H(U, s)$ (experimental result) and $H_{i}(U) \cdot D(s)$ (simulation) with $U=40 \mathrm{~V}$.

In order to confirm the previous conclusion, the same experiments and comparison were carried out with a sine 
voltage of an amplitude of $20 \mathrm{~V}$. As shown in Fig. 12, the equivalence $H(U, s)=H_{i}(U) \cdot D(s)$ is still obtained.
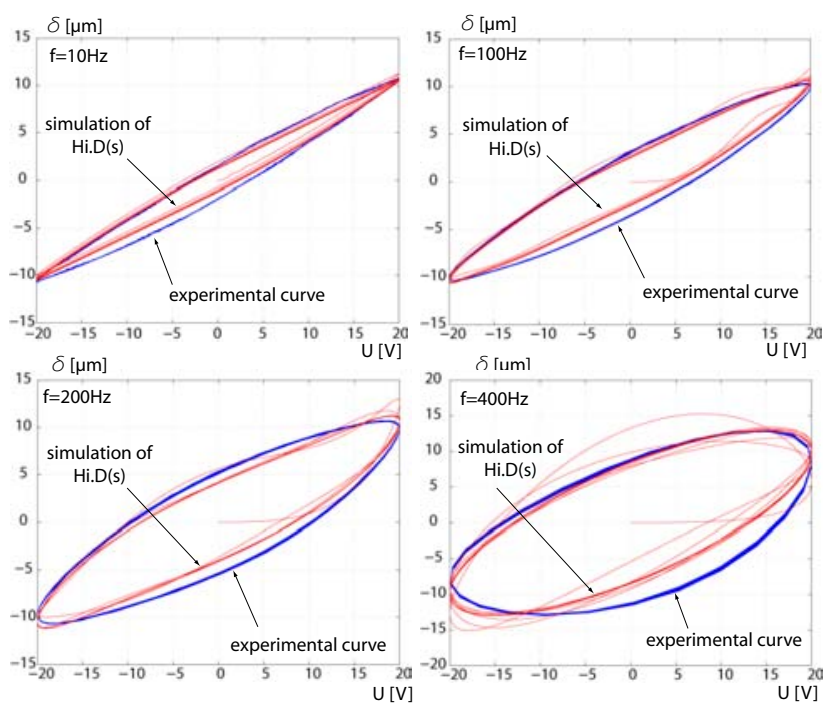

Fig. 12. Comparison of $H(U, s)$ (experimental result) and $H_{i}(U) \cdot D(s)$ (simulation) with $U=20 \mathrm{~V}$.

While the Bouc-Wen model used for the simulation is a valid approximation of the hysteresis behaviour of the piezocantilever, it contains nonlinear terms and consequently the synthesis of a linear controller is difficult. This is why we propose a new approximate model. The proposed model is linear but with uncertainty. While it is not intended to be implemented, it is used to synthesize a linear controller. In the new version,

\section{QUADRILATERAL APPROXIMATION AND IDENTIFICATION OF THE STATIC PART}

\section{A. Presentation of the quadrilateral approximation}

From the previous result and from (Eq. 3), we have:

$$
\delta=s_{p} \cdot D(s) \cdot F+H_{i}(U) \cdot D(s)+C_{r}(U, s)
$$

In this section, we are going to find a simple model linking the input voltage $U$ and the resulting deflection $\delta$. As a linear dynamic model is required to synthesize a linear controller, the term $H_{i}(U) \cdot D(s)$ is used to derive the model while the creep $C_{r}(U, s)$ and the mechanical terms $s_{p} \cdot D(s) \cdot F$ are considered as disturbances. In fact, the force $F$ is due to the contact with a manipulated object and then behaves like a disturbance. Next, the problem is to seek an approximate model of $H_{i}(U)$. For that, we propose a new approximation: the quadrilateral approximation. The principle is detailed below.

A nonlinear curve can be approximated by a piecewise affine function, which is called multilinear approximation. For example, a hysteresis curve can be represented by Fig. 13-a. Assuming that the hysteresis does not reach saturation, it can be sufficiently approximated by four segments (Fig. 13-b).

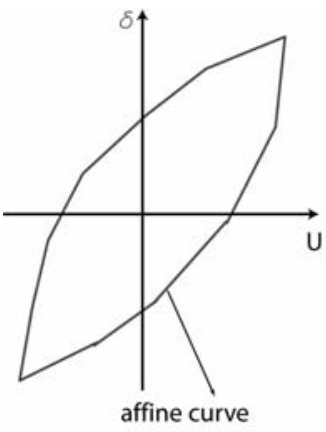

(a)

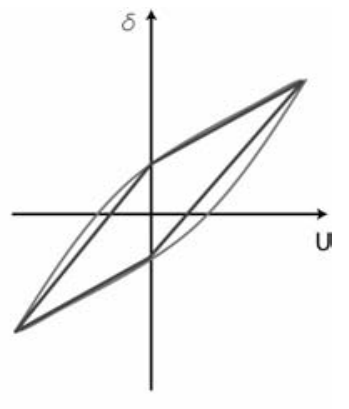

(b)
Fig. 13. a: multilinear approximation of the hysteresis. b: quadrilateral approximation of the hysteresis.

Let $\left(\Delta_{M}\right)$ and $\left(\Delta_{m}\right)$ represent the two straightlines of the quadrilateral with respectively the maximal and the minimal slopes:

$$
\begin{cases}\left(\Delta_{M}\right): & \delta(t)=\alpha_{M} \cdot U(t)+\delta_{M}(.) \\ \left(\Delta_{m}\right): & \delta(t)=\alpha_{m} \cdot U(t)+\delta_{m}(.)\end{cases}
$$

where $\alpha_{M}$ (respectively $\alpha_{m}$ ) represents the maximal (respectively minimal) slope and $\delta_{M}($.$) (respectively \delta_{m}($.$) )$ represents the corresponding offset.

Let $\alpha_{O}$ be the middle value of the maximal and the minimal slopes and $\alpha_{E}$ be their radius, such as:

$$
\left\{\begin{array}{l}
\alpha_{O}=\frac{\alpha_{M}+\alpha_{m}}{2} \\
\alpha_{E}=\frac{\alpha_{M}-\alpha_{m}}{2}
\end{array}\right.
$$

we propose to replace the static hysteresis $H_{i}(U)$ by a linear model with a nominal constant slope $\alpha_{O}$ and a new offset $\delta_{H}$ :

$$
H_{i}(U) \rightarrow \alpha_{O} \cdot U+\delta_{H}
$$

Thereby, the hysteresis of the real system has the following characteristics:

$$
\left\{\begin{array}{l}
H_{i}(U)=\alpha_{\text {real }} \cdot U+\delta_{H} \\
\alpha_{O}-\alpha_{E} \leq \alpha_{\text {real }} \leq \alpha_{O}+\alpha_{E}
\end{array}\right.
$$

While (Eq. 10) is used as a nominal model, the (Eq. 11) indicates that the nominal model has uncertainty relative to the real system. The uncertainty is refered to the nominal static gain $\alpha_{O}$ and its amplitude can be determined with the radius $\alpha_{E}$. Secondly, $\delta_{H}$ can be considered as a disturbance.

\section{B. Identification of the parameters}

To identify $\alpha_{O}$, a $(U, \delta)$-curve is plotted in order to obtain a hysteresis curve. For that, a sine voltage $U$ is applied. On the one hand, the frequency has been chosen to be small ( $f=$ $0.1 \mathrm{~Hz}$ ) in order to avoid the effect of the dynamic but should be higher than the creep cut-off frequency. On the other hand, the amplitude is chosen $(40 \mathrm{~V})$ to be the maximal range so that the identification is done with the external loop.

When the hysteresis curve derived from the experiments is obtained, a quadrilateral is fitted to it. The sides (straightlines) of the quadrilateral are chosen to connect the vertexes 
(obtained at $U=40$ ) and the middle points (obtained at $U=0$ ) of the hysteresis curve. At the ventral points, the hysteresis amplitude is maximal. The slope of each side of the quadrilateral is computed. After computing, the lowest slope is chosen to be $\alpha_{m}$ while the highest slope is $\alpha_{M}$. Fig. 14 presents the hysteresis curve and the two upper sides of the quadrilateral giving the values of $\alpha_{m}$ and $\alpha_{M}$.

Thereby, the nominal static gain $\alpha_{O}$ and the radius $\alpha_{E}$ are computed using the (Eq. 9). We obtain $\alpha_{O}=502 \times$ $10^{-9}[\mathrm{~m} / \mathrm{V}]$ and $\alpha_{E}=123 \times 10^{-9}[\mathrm{~m} / \mathrm{V}]$.

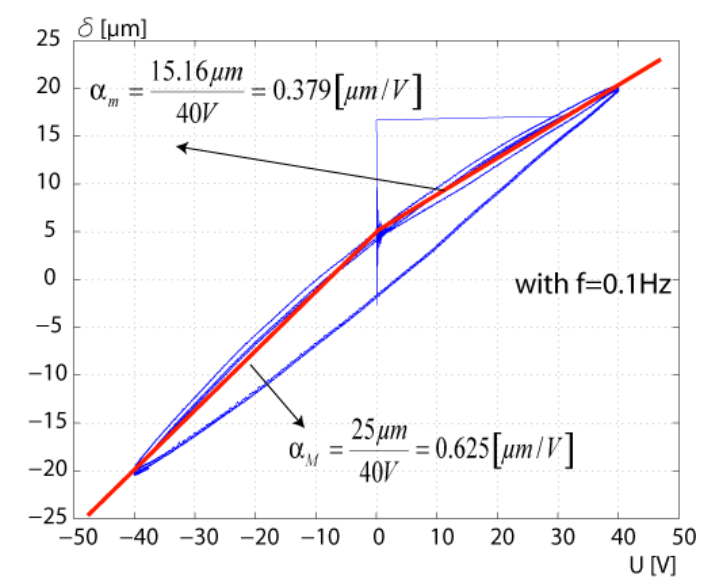

Fig. 14. Identification of the parameters.

\section{Performance of the identified model with a varying ampli- tude}

As we can see, the static nominal model is identified from the external loop of the static hysteresis, where the amplitude of the sine voltage is maximal $(U=40 \mathrm{~V})$. Here, we show that when the amplitude varies and internal loops are obtained, the radius $\alpha_{E}$ (and then the uncertainty) is lower than the one when the amplitude is maximal. Remember that $\alpha_{E}$ is given by (Eq. 9).

Since the model is calculated from the maximal and minimal slopes of the quadrilateral, the quadrilateral approximation of the internal loops should have slopes that are comprised in $\left[\alpha_{m}, \alpha_{M}\right]$. So, $\alpha_{E}$ with internal loops are still lower than $\alpha_{E}$ with the external loop. To check that, experiments with varying amplitude sine voltage were performed. The results show that the internal loops are slightly tilted relative to the external loop (Fig. 15). It can be seen that the maximal slopes $\alpha_{M}^{\text {internal }}$ of the internal loops are lower than the maximal slope $\alpha_{M}$ of the external loop. However, the minimal slope $\alpha_{m}$ of the external loop is higher than the minimal slopes $\alpha_{m}^{\text {internal }}$ of the internal loops. The minimal slopes are never null even if the amplitude of the sine voltage is very small. We have:

$$
\left\{\begin{array}{l}
\alpha_{O}<\alpha_{M}^{\text {internal }}<\alpha_{M} \\
0<\alpha_{m}^{\text {internal }}<\alpha_{m}<\alpha_{O}
\end{array}\right.
$$

In spite of that, the values of the minimal slopes are not too different from $\alpha_{m}$. So, it can be stated that:

$$
\alpha_{m}^{\text {internal }} \approx \alpha_{m}<\alpha_{O}<\alpha_{M}^{\text {internal }}<\alpha_{M}
$$

Then, according to the definitions in (Eq. 9) and (Eq. 11) and the result in (Eq. 13), we can construe that the error of the model on internal loops is lower than the error on the external loop.

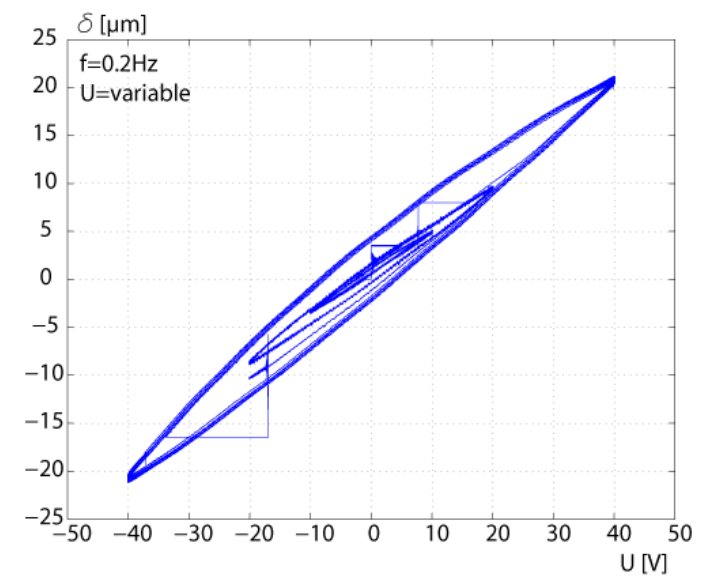

Fig. 15. Hysteresis curve with a varying amplitude and a constant frequency of input voltage.

\section{COMPlete MOdel}

Using the complete expression (Eq. 7) and the quadrilateral approximation (Eq. 10), we have:

$$
\delta=s_{p} \cdot D(s) \cdot F+\alpha_{O} \cdot D(s) \cdot U+\delta_{H}+C_{r}(U, s)
$$

The term $\alpha_{O} \cdot D(s) \cdot U$ is a dynamic linear relation between the deflection and the input voltage. It is of great interest to choose it as the nominal model. On the other hand, the force can be considered as a disturbance. Actually, it is due to the contact of the cantilever with a manipulated micro-object. Because the creep is a source of inacuracy in micromanipulation, it can also be considered as a disturbance. Despite the dependence of the creep on the input $U$, such consideration is possible because the creep $C_{r}(U, s)$ is slow and very small relative to the term $\alpha_{O} \cdot D(s) \cdot U$.

So, the nominal model of the piezocantilever is:

$\delta(s)=\frac{\alpha_{O}}{a \cdot s^{2}+b \cdot s+1} \cdot U(s)+\frac{1}{a \cdot s^{2}+b \cdot s+1} \cdot \delta_{0}(s)$

where:

- the dynamic part $D(s)$ has been replaced by the (Eq. 4),

- the nominal static gain $\alpha_{O}$ completed by an uncertainty represents the real static gain: $\alpha_{O}-\alpha_{E} \leq \alpha_{\text {real }} \leq \alpha_{O}+$ $\alpha_{E}$.

- and the disturbance is defined by:

$$
\delta_{0}=s_{p} \cdot F+\left(\delta_{H}+C_{r}(U, s)\right) \cdot D^{-1}(s)
$$

It can be stated that if the real system is linear, we have: 


$$
\left\{\begin{array}{c}
\alpha_{E}=0 \\
\delta_{H}=0
\end{array} \Leftrightarrow \alpha_{\text {real }}=\alpha_{O}\right.
$$

Fig. 16 represents the scheme of the nominal system.

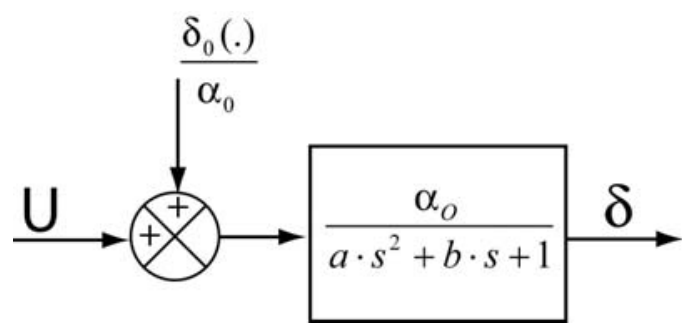

Fig. 16. Scheme of the nominal system.

Due to the presence of a disturbance (internal and external), a closed loop control is necessary. The choice of the controller is guided by the characteristics of the model and the specifications according to the micromanipulation tasks:

- the nominal static gain $\alpha_{O}$ is subjected to uncertainty,

- the interchange of piezocantilevers as seen in the first section of this paper is often required, so there may exist other uncertainty types in the model,

- as we can see in Fig. 9, the nominal model (second order) neglects fast dynamic. The higher order model fits the real system better than the nominal model,

- the effects of the environment (temperature, vibrations, etc.) must be rejected,

- finally, the performances required in micromanipulation should be taken into account.

To obtain robust stability and robust performances considering the different environmental disturbances and the characteristics of the proposed model, a robust controller best fits our objectives. For that, a $H_{\infty}$ controller has been synthesized. The performances are compared with those obtained with a PID controller.

\section{V. $H_{\infty}$ ROBUST CONTROL OF THE PIEZOCANTILEVER}

A $H_{\infty}$ robust controller is synthesized using the nominal model (Eq. 15). The results are compared with those of a PID-controller in order to show the robustness. As the controllers are intended to be implemented in an embedded computer, we design discrete controllers. Nevertheless, the best and simplest way to design a discrete $H_{\infty}$ based optimal controller is via bilinear transformation [27] [28] [29]. The main reasons are that continuous analysis is simpler, more standard and has a greater physical sense than the discrete analysis. For these reasons, we use the steps presented in Fig. 17 to synthesize the discrete $H_{\infty}$ controller. First, we derive a discret model from the (continuous) system in order to take into account the DAC-DCA and the sampling time of the computer. The transformation uses the zero-order-holder (zoh) method. Then, using this discrete model, we derive a pseudo-continuous model. A pseudo-continuous model is the transformation of a discrete model into a continuous model using the Tustin inverse bilinear transformation. It is called pseudo-continuous because it is an image of the initial continuous model using two transformations (the zoh and the Tustin bilinear transformations). In the pseudo-continuous domain, a controller can be synthesized. Finally, the discrete controller is obtained using the Tustin direct bilinear transformation of the synthesized controller. The Tustin bilinear transformation is defined as a bijective application $s=f(z)$, with $s$ the Laplace variable and $z$ the discrete operator, such as:

$$
s=\frac{2}{T_{s}} \cdot \frac{z-1}{z+1}
$$

with $T_{s}=0.2 m s$ the sampling time.

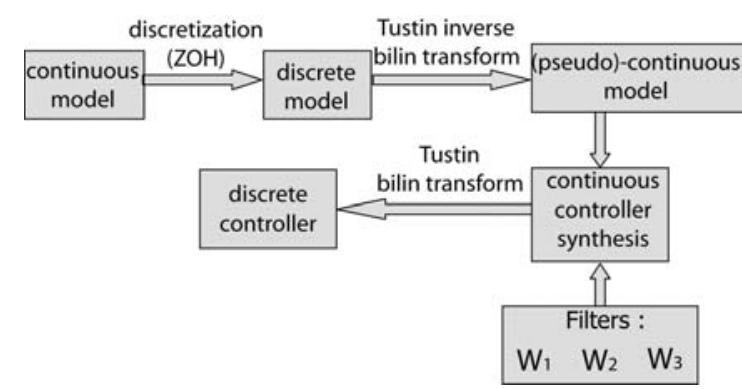

Fig. 17. The steps used to synthesize the discrete controller.

Fig. 18 shows the scheme of the closed-loop system in the pseudo-continuous domain. In this figure, $\delta_{c}$ represents the reference deflection. Two weighting functions are introduced: $W_{1}(s)$ for the tracking performances and $W_{2}(s)$ for the $\delta_{0}$ disturbance rejection.

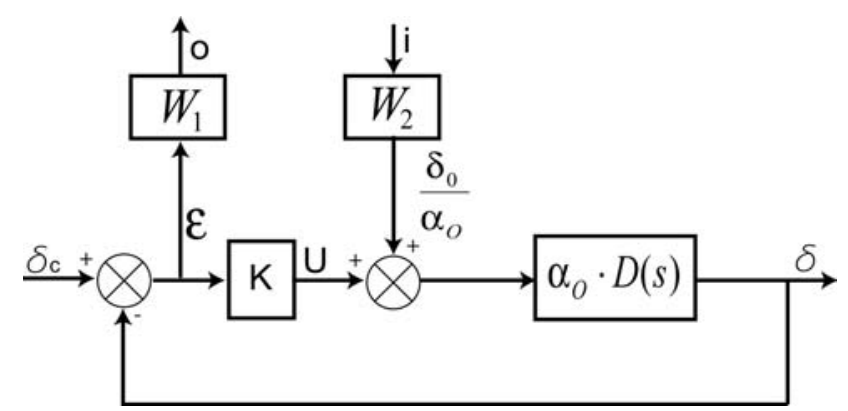

Fig. 18. The closed-loop scheme with the weighting transfer functions.

\section{A. Standard form}

Let $P(s)$ be the model equivalent to the nominal system $G(s)=\alpha_{O} \cdot D(s)$ augmented by the weighting functions. Fig. 19 represents the corresponding standard-form.

The standard $H_{\infty}$ problem consists in finding an optimal value $\gamma>0$ and a controller $K(s)$ stabilizing the closed-loop scheme of Fig. 19 and guaranteeing the following inequality [30]:

$$
\left\|F_{l}(P(s), K(s))\right\|_{\infty}<\gamma
$$




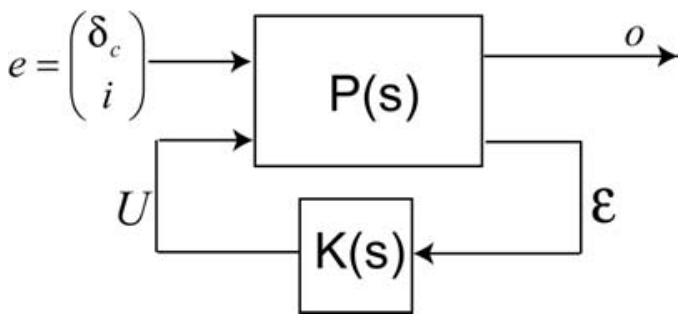

Fig. 19. The standard form.

where $F_{l}(.,$.$) is the lower Linear Fractionar Transformation$ and is defined by $F_{l}(P(s), K(s))=\frac{o(s)}{e(s)}$.

From Fig. 18, we have:

$$
o=W_{1} \cdot S \cdot \delta_{c}-W_{1} \cdot S \cdot G \cdot W_{2} \cdot i
$$

where $S=(1+K \cdot G)^{-1}$ is the sensitivity function.

Using the condition (Ineq. 19) and (Eq. 20), we infer:

$$
\begin{gathered}
\left\|W_{1} \cdot S\right\|_{\infty}<\gamma \\
\left\|W_{1} \cdot S \cdot G \cdot W_{2}\right\|_{\infty}<\gamma
\end{gathered} \Leftrightarrow \quad \begin{gathered}
|S|<\frac{\gamma}{\left|W_{1}\right|} \\
|S \cdot G|<\frac{\gamma}{\left|W_{1} \cdot W_{2}\right|}
\end{gathered}
$$

To solve the problem (Eq. 21), we use the Glover-Doyle algorithm which is based on the Riccati equations [31] [32]. The wanted performances are introduced through the weighting functions.

\section{B. Choice of the weighting functions}

The choice of the weighting functions is derived from the specifications. The latter have been chosen from general needs in micromanipulation.

1) Choice of $W_{1}$ : the transfer function $\frac{1}{W_{1}}$ is chosen from the specifications on the tracking performances. These specifications are:

- the maximal response time is lower than $10 \mathrm{~ms}$,

- the overshoot is null,

- and the maximal statical error is lower than $0.1 \%$.

For this, we choose:

$$
\frac{1}{W_{1}}=10^{-3} \cdot \frac{3 \cdot s+1}{0.003 \cdot s+1}
$$

so:

$$
W_{1}=10^{3} \cdot \frac{0.003 \cdot s+1}{3 \cdot s+1}
$$

2) Choice of $W_{2}$ : here, the specifications relative to the $\delta_{0}$ disturbance rejection are used. In the (Eq. 21), the transfer between the output and the disturbance is defined as follows:

$$
\frac{\delta \cdot \alpha_{O}}{\delta_{0}}=S \cdot G
$$

Replacing $\delta_{0}$ by the (Eq. 16) and working in the static mode $(s=0)$, we have:

$$
\frac{\delta \cdot \alpha_{O}}{\delta_{0}}=\frac{\delta \cdot \alpha_{O}}{s_{p} \cdot F+\delta_{H}+C_{r}(U, s)}
$$

The previous equation quantifies the influence of the force $F$, the hysteresis offset $\delta_{H}$ and the creep $C_{r}(U, s)$ on the deflection $\delta$ in the closed-loop form. Our objective is as follows: when $F=5 m N, \delta_{H}=5 \mu m$ and $C_{r}(U, s)=5 \mu m$, the deflection is less than $\delta=1 \mu \mathrm{m}$. These values respectively correspond to:

- the maximal force used to manipulate a micro-object,

- the offset of the quadrilateral segments of the external hysteresis loop obtained with $U=40 \mathrm{~V}$,

- the creep static value obtained with $U=40 \mathrm{~V}$.

We have:

$$
S \cdot G(s=0)=0.25 \times 10^{-7}
$$

This is the characteristic in the static mode and should be lower than $\frac{\gamma}{W_{1} \cdot W_{2}}(s=0)$. In addition, we specify a cut-off frequency of $\frac{1}{W_{1} \cdot W_{2}}$ at $4000[\mathrm{rad} / \mathrm{s}]$ in order to ensure the rejection of a wide range of frequency disturbances. Thus, we choose the following nominal performances:

$$
\frac{1}{W_{1} \cdot W_{2}}=0.25 \times 10^{-7} \cdot \frac{10^{-1} \cdot s+1}{\frac{1}{4000} \cdot s+1}
$$

We infer:

$$
W_{2}=4 \times 10^{4} \cdot \frac{\left(\frac{1}{4000} \cdot s+1\right) \cdot(3 \cdot s+1)}{\left(10^{-1} \cdot s+1\right) \cdot(0.003 \cdot s+1)}
$$

\section{Calculation of the controller}

The pseudo-continous controller has been calculated with the Matlab software and its Robust Control Toolbox. Afterwards, it has been transformed into a discrete controller using (Eq. 18). We have:

$$
K=\frac{\begin{array}{c}
3.9 \times 10^{5} \cdot z^{5}-9.1 \times 10^{5} \cdot z^{4}+5.3 \times 10^{5} \cdot z^{3} \\
+5.4 \times 10^{5} \cdot z^{2}-9.2 \times 10^{5} \cdot z+3.8 \times 10^{5}
\end{array}}{z^{5}-1.1 \cdot z^{4}-1.6 \cdot z^{3}+1.9 \cdot z^{2}+0.6 \cdot z-0.8}
$$

and the optimal value of $\gamma$ is:

$$
\gamma_{o p t}=1.05
$$

\section{Experimental results}

(Eq. 29) has been implemented into the Simulink software and run on the DSpace board such as the sampling time is $T s=0.2 \mathrm{~ms}$. First, a step reference, from $-25 \mu \mathrm{m}$ to $25 \mu \mathrm{m}$, is applied. Fig. 20 presents the results. It clearly shows that the wanted performances are obtained, i.e. response time and overshoot.

To evaluate more precisely the performances, harmonic analysis has been performed. The experimental magnitude is then presented in Fig. 21. The results indicate that the bandpass is $300 \mathrm{rad} / \mathrm{s}$, it corresponds to a response time nearly equal to $10 \mathrm{~ms}$. As there is no resonance, it can be stated that no overshoot and no vibration will appear in the step response.

In order to evaluate the robustness margins of the closed loop system, the Black-Nichols diagram is plotted (Fig. 22). 


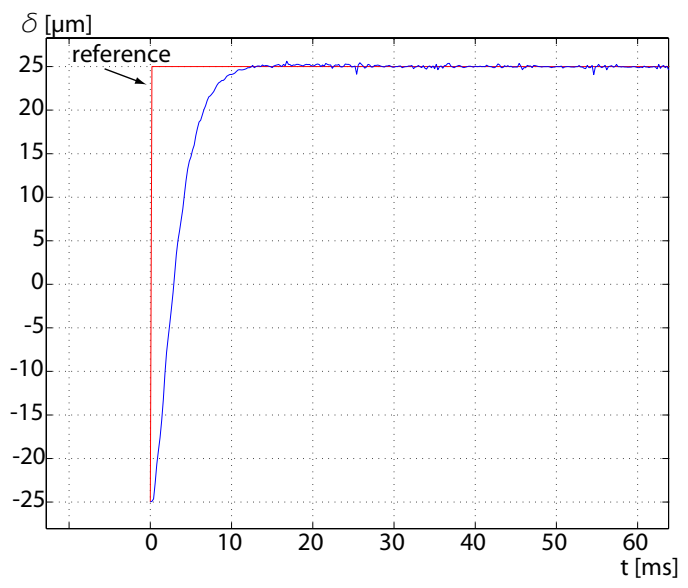

Fig. 20. Step response of the closed-loop system.

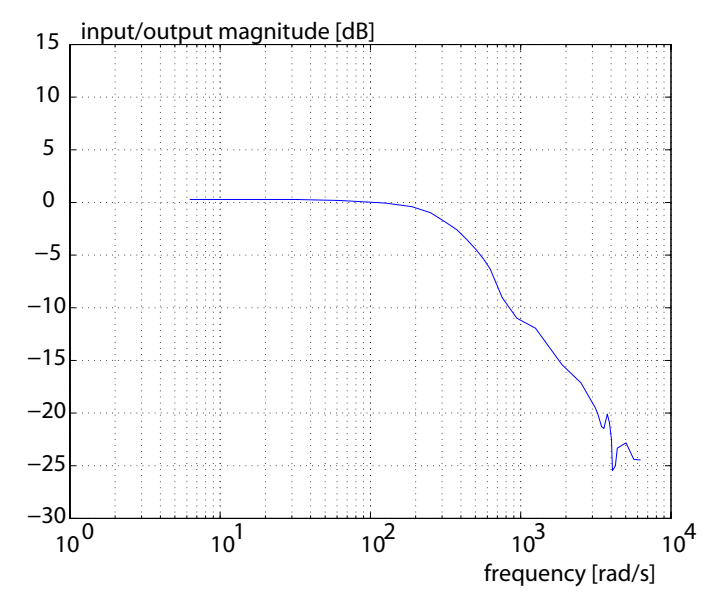

Fig. 21. Harmonic experiments of the closed-loop system.

TABLE II

Robustness margins.

\begin{tabular}{|l|l|}
\hline \hline Gain margin (dB) & Phase margin $\mathbf{(}^{\circ}$ ) \\
\hline$]-\infty, 35]$ & $87($ at $341 \mathrm{rad} / \mathrm{s})$ \\
\hline \hline
\end{tabular}

The margin gain and the margin phase can be deduced from the figure. We have:

From the margin gain, it can be deduced that the stability of the closed loop system is ensured as long as the gain $\alpha_{\text {real }}$ is between 0 and $1.75 \times \alpha_{O}=878.5 \times 10^{-9}\left[\frac{m}{V}\right]$. This range includes the identified gains $\alpha_{m}$ and $\alpha_{M}$ that characterizes the quadrilateral (see Fig. 14). That theoretically indicates that the stability is ensured in the applied range of voltage.

\section{CONCLUSION}

This paper presents a new method to model a nonlinear piezoelectric cantilever and presents the design of a $H_{\infty}$ controller to control it.

First, we have demonstrated that the dynamic hysteresis of the piezocantilever is equivalent to a static hysteresis, a varying gain, followed by a linear dynamic system. Then, we have proposed a new approximate model of the static hysteresis.

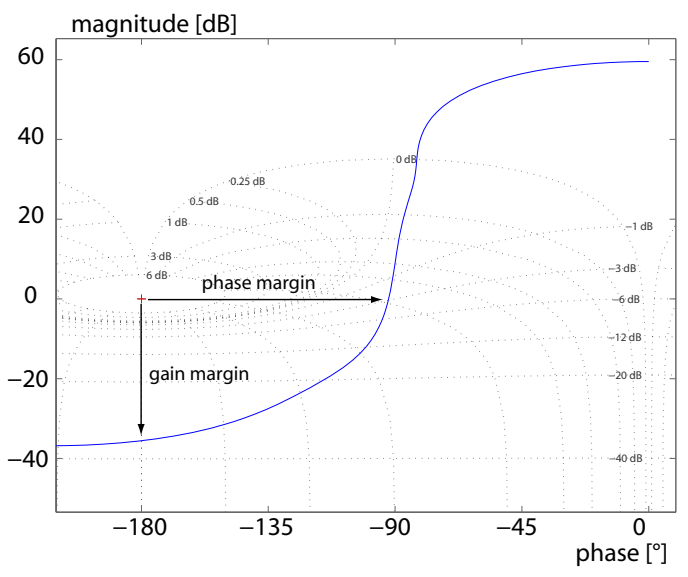

Fig. 22. Black-Nichols diagram.

It consists in approximate it by a quadrilateral characterized by the average, the maximal and the minimal slopes and the offset of the different straights. While the average slope is considered as a nominal gain, the offset of the straight have been used to synthesize a $H_{\infty}$ controller. On the other hand, the creep and the external applied force have been considered as a disturbance and their effect were taken into account during the controller synthesis. Finally, experiments have been carried out and clearly show the robustness of the synthesized $H_{\infty}$ controller relative to the context of the micromanipulation.

\section{ACKNOWLEDGMENT}

This project is partially supported by the EUPASSproject (Evolvable Ultra-Precision Assembly SystemS : http://eupass.org/).

\section{REFERENCES}

[1] D. L. Heiserman, 'Piezoelectric polymer micromanipulator', United States Patent, $N^{o}$ US 4,610,475, 1986.

[2] Y. Haddab, N. Chaillet and A. Bourjault, 'A microgripper using smart piezoelectric actuators', Proc. of IEEE/RSJ International Conference on Intelligent Robot and Systems - IROS, Takamatsu - Japan, 2000.

[3] J. Agnus, J. M. Breguet, N. Chaillet, O. Cois, P. de Lit, A. Ferreira, P. Melchior, C. Pellet and J. Sabatier, 'A smart microrobot on chip: design, identification and modeling', Proc. IEEE/ASME International Conference on Advanced Intelligent Mechatronics - AIM, Kobe - Japan, pp.685-690 2003.

[4] J. Zhong, S. Seelecke, R. C. Smith and C. Büskens, 'Optimal control of piezoceramic actuators'.

[5] J. A. Main, E. Garcia and D. V. Newton, 'Precision position control of piezoelectric actuators using charge feedback' Journal of guidance, Control and Dynamics, 18(5):1068-1073, 1995.

[6] H. Jung, J.Y. Shim and D. Gweon, 'New open-loop actuating method of piezoelectric actuators for removing hysteresis and creep', Review of Scientific Instruments 71 (9): 3436-3440 SEP 2000.

[7] K. Kuhnen and H. Janocha, 'Modeling of transfer characteristic of piezoelectric transducers by creep and hysteresis operators'.

[8] D. Croft, G. Shed and S. Devasia, 'Creep, hysteresis and vibration compensation for piezoactuators:atomic force microscopy application', ASME Journal of dynamic systems, measurement and control, vol.123, pp35-43, 2001.

[9] P. Ge and M. Jouaneh, 'Generalised preisach model for hysteresis nonlinearity of piezoelectric actuators', Precision Engineering, 20, 99$111,1997$.

[10] D. Hughes and J. T. Wen, 'Preisach modeling of piezoceramic and shape memory alloy hysteresys', Smart Mater. Struct., pp.287-300, 1997. 
[11] R. B. Mrad and H. Hu, 'A model for voltage to displacement dynamics in piezoceramic actuators subject to dynamic-voltage excitations', IEEE Transactions on mechatronics 7(4), 479-489 2002.

[12] R. C. Smith and Z. Ounaies, 'A hysteresis model for piezoceramic materials', NASA-ICASE Report No99-229 August 1999.

[13] Y. Gao and S. Tse, 'Modelling of piezoelectric actuator based nanopositioning system under sinusoidal excitation using multi-polynomial regression', Journal of Physics: Conference Series 13, pp.98-101, 2005.

[14] M. Goldfarb and N. Celanovic, 'A lumped parameter electromechanical model for describing the nonlinear behavior of piezoelectric actuators', ASME Journal of dynamc systems, measurement and control, 119:478485, 1997.

[15] S. Lining, R. Changhai, R. Weibin, C. Liguo and K. Minxiu, 'Tracking control of piezoelectric actuator based on a new mathematical model', Journal in Micromech. Microeng. 14 pp1439-1444, August 2004.

[16] T. S. Low and W. Guo, 'Modeling of a three-layer piezoelectric bimorph beam with hysteresis', J. Microelectromech. Syst., vol.4, pp230-237, 1995.

[17] C. W. Wong, Y. Q. Ni and J. M. Ko, 'Steady state oscillation of hysteretic differential model. i-response analysis', J. Engineering Mechanics, 120, 1994.

[18] S. Chonan, Z. Jiang and T. Yamamoto, 'Nonlinear hysteresis compensation of piezoelectric ceramic actuators', Journal. Intell. Mater. Syst. Struct. pp.150-156, 1996.

[19] M. S. Tsai and J. S. Chen, 'Robust tracking control of a piezoactuator using a new approximate hysteresis model', Transactions of the ASME Journal of Dynamic Systems, Measurement and Control, 125, pp.96-102, 2003.

[20] J. A. Main and E. Garcia, 'Piezoelectric stack actuators and control system design: strategies and pitfalls', Journal. Guid. Control Dyn. 20(3), pp.479-485, 1997.

[21] D. Croft and S. Devasia, 'Hysteresis and vibration compensation for piezoactuators', Journal. Guid. Control Dyn. 21, pp.710-717, 1998.

[22] S. Devasia, E. E. Eleftheriou, R. Moheimani, 'A survey of control issues in nanopositioning' IEEE Transactions on Control System Technology, Vol.15, $N^{o} 15$, pp.802-823, 2007.

[23] I. D. Mayergoyz, 'Mathematical models of hysteresis and their applications' Amsterdam, Boston: Elsevier, 2003.

[24] IEEE Standard on Piezoelectricity. Institute of Electrical and Electronics Engineers. Std 176-1978, september 1978.

[25] J. G. Smits and W. S. Choi, 'The constituent equations of piezoelectric heterogeneous bimorphs', IEEE Transactions on Ultrasonics, Ferroelectrics and Frequency Control, Vol.38, $N^{\circ} 38$, May 1991.

[26] J. L. Pons, 'Emerging actuator technologies: a micromechatronic approach', ISBN 0-470-09197-5, Wiley, 2005.

[27] T. Chen and B. A. Francis, 'Optimal sampled-data control systems', Springer-Verlag, London.

[28] K. Zhou, J. C. Doyle and K. Glover, 'Robust and optimal control', Prentice-Hall, Englewood Cliffs, NJ.

[29] L. Mirkin, 'On discrete-time $H^{\infty}$ problem with a strictly proper controller', International Journal of Control 66, $N^{\circ} 5$, pp.747-765, 1997.

[30] G. J. Balas, J. C. Doyle, K. Glover, A. Packard and R. Smith, 't-analysis and synthesis toolbox', The Mathworks User's Guide-3, 2001.

[31] K. Glover and J. C. Doyle, 'State-space formulae for all stabilizing controllers that satisfy an $H_{\infty}$-norm bound and relations to risk sensivity', Systems \& Control Letters, vol.11, pp.167-172, 1988.

[32] J. C. Doyle, K. Glover, P. K. Khargonekar and B. A. Francis, 'Statespace solutions to standard $H_{2}$ and $H_{\infty}$ control problems', IEEE Transaction Automatic Control, AC $34 N^{\circ} 8$, pp.831-846, 1989.

[33] B. C. Moore, 'Principal component analysis in linear systems: controllability, observability and model reduction', IEEE Transaction on Automatic Control, AC-26(1): 17-32, 1981.

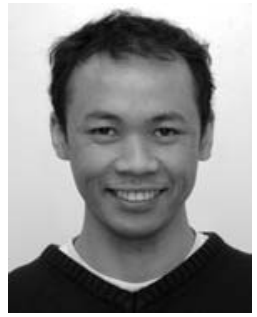

Micky Rakotondrabe: engineering diploma from the Institut Supérieur de Technologie du Nord (ISTN Lille France) in 2002, DEA diploma (MSc equivalent) in Automatic Control from the Institut National des Sciences Appliquées (INSA Lyon France) in 2003, PhD degree in Automatic Control from the Université de Franche-Comté (Besançon France) in 2006. Since September 2007, he is an Associate Professor at the Université de Franche-Comté and a researcher at the FEMTO-st Institute. His field of research concerns the Control Theory applied to microsystems and microrobots. He has also contributed to the design, the development and control of microfactory during his $\mathrm{PhD}$ thesis.

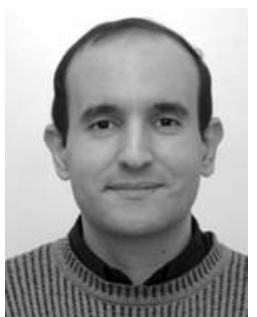

Yassine Haddab received the Engineering degree in electrical engineering at the University of TiziOuzou, Algeria, the M.S. degree at ENSMM, Besançon, France and the Ph.D. degree in 2000 at the University of Franche-Comté, Besançon, France. His work dealt with the design, the modelling and the control of micromanipulation systems. Since 2002 he is an Associate Professor at ENSMM Besançon where he teaches control theory and microrobotics. His research interests are the design and control of high precision microrobots and microsystems. $\mathrm{He}$ also contributes to the development of microfactory concepts and new microrobots architectures.

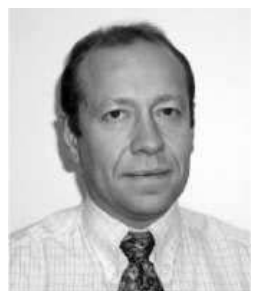

Philippe Lutz joined the University of FrancheComté in Besancon as Professor in 2002. He is currently the head of the research group 'Automated Systems for Micromanipulation and Microassembly' of the AS2M department of FEMTO-ST. His research activities are focussed on the design and the control of MicroMechatronic Systems. He teaches at the master level the design of Mechatronic Systems and Production System. Engineer of the ENSMM in 1990 and Doctor of the University of Franche-Comté in Automation and Computer Science in 1994, he was Associate Professor in the INSA of Strasbourg since 1994 until 2002. During this period, he has led the Mechatronics activities and developed research on inventive theory for solving problems. 